Research Article

\title{
Data Analysis of Physical Fitness Monitoring Based on Mathematical Models
}

\author{
Cuixiang Guo $\mathbb{D},{ }^{1}$ Junwu Suo $\mathbb{D},{ }^{2}$ Chunguang Xu, ${ }^{1}$ Xinhua Yang, ${ }^{1}$ and Liping Zhang ${ }^{1}$ \\ ${ }^{1}$ Shandong Polytechnic, Jinan 250104, China \\ ${ }^{2}$ Shandong Jianzhu University, Jinan 250101, China \\ Correspondence should be addressed to Cuixiang Guo; guocuixiang@sdp.edu.cn and Junwu Suo; suojunwu@sdjzu.edu.cn
}

Received 22 May 2021; Revised 5 June 2021; Accepted 9 June 2021; Published 22 June 2021

Academic Editor: Gengxin Sun

Copyright ( $\odot 2021$ Cuixiang Guo et al. This is an open access article distributed under the Creative Commons Attribution License, which permits unrestricted use, distribution, and reproduction in any medium, provided the original work is properly cited.

Physical monitoring and analysis are of great significance to improve national physical fitness. The main work of this paper is that the physical health level of college students is studied and analysed by using a statistical model and mathematical model. According to the characteristics of the collected data, different mathematical models are established. Firstly, the grey correlation model is used to analyse the correlation between pull-up and other physical fitness indexes. Then, based on the classification of college students and the influence and flow law of interclass crowd, a differential equation system is established based on the LMC model. By analysing the existence and stability of the equilibrium point of the system, as well as the possible folding bifurcation and backward bifurcation at the equilibrium point, this paper makes qualitative and quantitative research on the trend of college students' physical exercise on campus.

\section{Introduction}

Relying on data analysis technology and mathematical model to improve the development of national fitness monitoring can give full play to the application of data analysis technology in the field of economic and social life and effectively improve the information technology level of national fitness monitoring, so as to provide objective and scientific data support for the improvement of national fitness. College students have the characteristics of concentration and easy management, which is the breakthrough to effectively improve the national physical quality. However, what are the factors that affect college students' physical exercise and how to improve their participation in physical exercise are of great significance in improving the national physical quality. In the research of college students' physique, how to improve the number of college students participating in physical exercise is a problem to be solved. The study found that college students do not participate in or less participate in physical exercise activities, not because of lack of time, but with receiving sports information media publicity. And the publicity information mainly comes from regular classroom learning and through interaction between students.

The earliest physical monitoring can be traced back to the 1860s. In the United States, Hitchcock M. D. took the lead in making annual periodic measurements of all students' age, height, weight, length, circumference, vital capacity, and muscle strength in 1861. In 1954, the Kraus-Weber test, which involved six minimum levels of strength and flexibility of low back muscles, was developed and tested on American and European children. Subsequently, the National Federation of health, sports, rehabilitation, and dance designed a physical fitness test method covering seven indicators, including $50 \mathrm{~m}$ running, $600 \mathrm{~m}$ running, standing long jump, softball throwing, round-trip running, pull-up, and sit-ups.

At present, the research on the monitoring and measurement of college students' physique data mainly includes the following methods: the physical fitness level of college students is studied by using the methods of literature, questionnaire interview, physical test, and street survey [1-3], the influencing factors of college students' physique are explored by using correlation analysis and high-tech 
instruments [4, 5], improving the model of college students' physique score $[6,7]$, combining machine-learning algorithm to predict college students' performance in body measurement [8-10]. Although these studies show the physical condition of college students from many fields, they can also classify and predict the physical condition of college students, but they cannot reflect the dynamic change process of college students' physical fitness behavior.

In order to improve the level of college students' daily physical exercise, researchers also studied the behavior and motivation of daily physical exercise. Bellar et al. [11] explored the level of college students' sports activities through a cross-sectional online survey. Feng et al. [12] used the generalized estimation model to analyse the influence of fitness consciousness and fitness behavior on college students' physique and found that strengthening daily exercise consciousness can effectively improve college students' physique level.

In order to find the hidden law of things, propagation dynamics $[13,14]$ can be used to build more realistic models. Different from the traditional statistical method, Choszcz et al. [15] applied population dynamics to sports activities, classified the objects into three categories, and established the mathematical model of human fixed group and nonfixed group to carry out competitive sports. Posluszny et al. [16] made a qualitative analysis of the fixed group model and gave the ordinary differential equation model and partial differential equation model of the nonfixed group. Wang et al. [17] proposed a group competitive sports activity model with a delay-dependent effect; after the qualitative research on the model, it is found that the number of times of college students participating in exercise every week and the time of each exercise are low.

Although the existing research shows the physical condition of college students from many aspects and it can also classify and predict the physique of college students, it cannot reflect the dynamic process of sports behavior. We found that according to different classification criteria, such as weekly exercise frequency, time, or place of each exercise, college students could be classified. Therefore, this paper classifies college students according to exercise frequency. Secondly, the will of physical exercise mainly comes from classroom learning and peer communication, based on the rule that the consciousness of physical exercise is affected; the linear and standard influence rates are used to establish the propagation dynamics model of sports population flow. Compared with the traditional statistical method, the dynamic method can make us understand the overall situation better from the flow direction of college students. By combining with computer simulation and other methods, we can have a more indepth and comprehensive understanding of the changing trend of college students' participation in physical exercise.

\section{Analysis of College Students' Pull-Up Performance Based on Grey Correlation Model}

As a sport to test the development level of human upper limb muscle strength, pull-up has been accepted as a physical fitness test item in many countries. The data of this paper comes from the physical fitness test results of 7921 male students in a university in China in 2019. Physical fitness test indexes include pull-up (times $/ \mathrm{min}$ ), BMI $\left(\mathrm{kg} / \mathrm{m}^{2}\right)$, vital capacity $(\mathrm{ml}), 50$ meters $(\mathrm{s})$, standing long jump $(\mathrm{cm})$, sitting forward $(\mathrm{cm})$, and 1000 meters $(\mathrm{s})$.

Statistical analysis shows that the distribution of each variable follows a normal distribution. $3 \sigma$ method is used to preprocess the data, and the outliers caused by improper records are removed. The basic information of the sample is shown in Table 1.

2.1. Correlation Analysis between Pull-Up and Other Indexes. Pearson correlation analysis [18] is often used to mine the correlation between variables in statistics to describe the linear correlation between variables. Pearson correlation coefficient can be used to describe the close relationship between two distance variables. The calculation formula is as follows:

$$
r=\frac{\sum_{i=1}^{n}\left(x_{i}-\bar{x}\right)\left(y_{i}-\bar{y}\right)}{\sqrt{\sum_{i=1}^{n}\left(x_{i}-\bar{x}\right)^{2}\left(y_{i}-\bar{y}\right)^{2}}},
$$

where $\bar{x}$ and $\bar{y}$ represent average values of two samples and $r$ is Pearson correlation coefficient; the larger the value, the higher the linear correlation between variables.

According to the sample data set, the Pearson correlation coefficient between the pull-up and each measurement index is shown in Table 2.

Table 2 shows that there is a significant correlation between pull-up and various physical measurement indexes, in which standing long jump and sitting forward are positively correlated with pull-up, and other indexes are negatively correlated with pull-up. BMI, standing long jump, and 1000 meters are highly correlated with pull-up, but the values of $r$ are all lower than 0.5 , so it is necessary to seek other methods to verify the correlation.

Correlation analysis is the comparison of the geometric relationship of the statistical data. The closer the geometric curve of the sequence is, the greater the correlation degree is. In this paper, the improved grey slope correlation degree is chosen to describe the correlation between pull-up and other indexes. The improved grey slope correlation degree can reflect the positive and negative of sequence correlation and eliminate the influence of dimensionless transformation of the original sequence on the correlation coefficient and correlation degree value. The calculation steps of the improved slope correlation degree method are as follows: 
TABLE 1: The basic information of body measurement sample.

\begin{tabular}{|c|c|c|c|c|c|c|c|}
\hline Index & BMI & Vital capacity & 50 meters & Standing long jump & Sitting forward & 1000 meters & Pull-up \\
\hline Maximum value & 14.31 & 4216 & 20 & 298 & 38.7 & 438 & 57 \\
\hline Minimum value & 41.28 & 552 & 5.25 & 128 & 11.9 & 126 & 0 \\
\hline Average value & 21.92 & 4009.2 & 7.83 & 227.3 & 20.6 & 251.9 & 5.8 \\
\hline
\end{tabular}

TABLe 2: Pearson correlation coefficient between pull-up and each measurement index.

\begin{tabular}{lcccccc}
\hline & BMI & Vital capacity & 50 meters & Standing long jump & Sitting forward & 1000 meters \\
\hline$r$ & $-0.336^{* *}$ & $-0.092^{* *}$ & $-0.153^{* *}$ & $0.308^{* *}$ & $0.112^{* *}$ & $-0.276^{* *}$ \\
Significance & 0.000 & 0.000 & 0.000 & 0.000 & 0.000 & 0.000 \\
\hline
\end{tabular}

${ }^{* *}$ Significant correlation at 0.01 level.

Step 1: dimensionless transformations of reference sequence $s_{0}=\left\{s_{0}(1), s_{0}(2), \ldots, s_{0}(n)\right\}$ and comparison sequence $s_{i}=\left\{s_{i}(1), s_{i}(2), \ldots, s_{i}(n)\right\}$ are carried out:

$$
y_{i}(k)=\frac{s_{i}(k)}{s_{i}(1)}, \quad i=0, \ldots, m ; k=1, \ldots, n \text {. }
$$

The slope of each time point is calculated as follows:

$$
\Delta y_{i}(k)=y_{i}(k+1)-y_{i}(k) \text {. }
$$

Then, the grey slope correlation coefficient of each time point is

$$
\varepsilon_{i}(k)=\operatorname{sgn}\left(\Delta y_{0}(k), \Delta y_{i}(k)\right) \cdot \frac{1+\left|\left(\Delta y_{0}(k) / \overline{\bar{y}_{0}}\right)\right|}{1+\left|\left(\Delta y_{0}(k) / \overline{\bar{y}}_{0}\right)\right|+\left|\left(\Delta y_{0}(k) / \overline{\bar{y}}_{0}\right)-\left(\Delta y_{i}(k) / \overline{\bar{y}_{i}}\right)\right|}
$$

where $\quad \bar{y}_{0}=(1 / n) \sum_{k=1}^{n} y_{0}(k), \quad \bar{y}_{i}=(1 / n) \sum_{k=1}^{n} y_{i}(k)$, and $\operatorname{sgn}\left(\Delta y_{0}(k), \Delta y_{i}(k)\right)$ is used to describe the positive and negative correlation of variables, when $\Delta y_{0}(k) \cdot \Delta y_{i}(k) \geq 0$. Its value is equal to 1 ; otherwise, its value is -1 .

Step 2: by calculating the grey correlation degree and sorting, the correlation between the comparison sequence and the reference sequence can be obtained:

$$
\gamma_{i}=\frac{1}{n-1} \sum_{k=1}^{n-1} \varepsilon_{i}(k)
$$

where $\gamma_{i}$ represents the correlation strength of index $i$.

The sampling data is divided according to the enrollment year, and the mean value of the physical measurement data of the students' enrollment in the same year is calculated to obtain a group of time series of the enrollment year from 2012 to 2020, shown as Table 3 .

Taking pull-up as the reference sequence and the other six indexes as the comparison sequence, according to the above steps, the correlation coefficient and correlation degree between the comparison sequence and the reference sequence are calculated, and the results are shown in Tables 4 and 5 .

2.2. Prediction of Pull-Up Performance Trend. Grey prediction [19] refers to establishing the corresponding differential equation by mining the rules of the system change through the processing of the original data, so as to predict future development. From the Pearson correlation coefficient, it can be seen that the linear correlation between each variable and pull-up is not strong, so it is impossible to establish the regression model with standard accuracy. Therefore, the grey prediction model with small sample demand is selected to predict the development trend of college students' pull-up performance in the future. In this paper, the data of the first eight years from 2012 to 2020 are selected to establish the model, the data of the ninth year are used to test the prediction accuracy of the model, and the optimal prediction model is obtained by optimizing the initial value and background value.

In the process of model construction, firstly, $y_{t}$ is calculated as an accumulation sequence of the original sequence $x_{t}$ :

$$
Y_{t}=\sum_{k=1}^{t} X_{t}, \quad t=1,2, \ldots, n .
$$

Then, a weighted adjacent value sequence $Z_{t}$ is generated as follows:

$$
Z_{t}=\vartheta Y_{t}+(1-\vartheta) Y_{t-1}, \quad 0<\vartheta<1
$$

When traditional $\operatorname{GM}(1,1)$ is used, $\vartheta=0.5$, the mean value sequence $Z_{t}=0.5\left(Y_{t}+Y_{t-1}\right)$.

The constructed model is defined as follows: 
TABLe 3: The data of students' enrollment in 1992-2000.

\begin{tabular}{lccccccc}
\hline Year & BMI & Vital capacity & 50 meters & Standing long jump & Sitting forward & 1000 meters & Pull-up \\
\hline 2012 & 21.68 & 4142.8 & 7.47 & 7.81 & 232.8 & 21.6 & 245.5 \\
2013 & 22.09 & 4152.1 & 7.92 & 228.6 & 2.4 & 249.5 & 5.9 \\
2014 & 22.12 & 4094.2 & 7.87 & 228.3 & 20.5 & 250.2 \\
2015 & 21.91 & 4133.9 & 7.82 & 228.5 & 19.8 & 252.6 \\
2016 & 21.98 & 4032.8 & 7.85 & 227.5 & 18.9 & 252.7 \\
2017 & 21.87 & 3982.7 & 7.89 & 224.8 & 18.6 & 252.9 \\
2018 & 21.83 & 3996.2 & 2.94 & 221.4 & 17.7 & 252.1 \\
2019 & 21.81 & 4048.6 & 8.02 & & 19.9 & 249.3 \\
2020 & 21.62 & 4067.9 & & & 4.6 & 3.6 \\
\hline
\end{tabular}

TABLE 4: Grey correlation coefficient between pull-up and each measurement index.

\begin{tabular}{lccccc}
\hline Correlation coefficient & $\varepsilon_{1}(k)$ & $\varepsilon_{2}(k)$ & $\varepsilon_{3}(k)$ & $\varepsilon_{4}(k)$ & $\varepsilon_{5}(k)$ \\
\hline$k=1$ & -0.9252 & -0.9407 & 0.9536 & 0.9513 & -0.9262 \\
$k=2$ & -0.9012 & 0.9116 & -0.8931 & -0.8977 & 0.9173 \\
$k=3$ & 0.9501 & -0.9378 & 0.9519 & 0.9498 & 0.9872 \\
$k=4$ & 0.9807 & 0.9946 & 0.9831 & -0.9762 & 0.9971 \\
$k=5$ & 0.9452 & 0.9501 & -0.9412 & 0.9477 & 0.9821 \\
$k=6$ & -0.9222 & -0.9205 & -0.9163 & 0.9332 & -0.9225 \\
$k=7$ & 0.9045 & -0.8932 & -0.8951 & 0.9162 & -0.9776 \\
$k=8$ & 0.9478 & -0.9359 & -0.9301 & -0.9327 & -0.9418 \\
\hline
\end{tabular}

TABLE 5: The correlation degree and ranking of pull-up and various physical measurement indexes.

\begin{tabular}{lcccccc}
\hline & $\gamma_{1}$ & $\gamma_{2}$ & $\gamma_{3}$ & $\gamma_{4}$ & $\gamma_{5}$ & $\gamma_{6}$ \\
\hline $\begin{array}{l}\text { Correlation } \\
\text { degree }\end{array}$ & 0.2473 & 0.2212 & -0.2114 & 0.2265 & 0.2638 & -0.4017 \\
Ranking & 3 & 5 & 6 & 4 & 2 & 1 \\
\hline
\end{tabular}

$$
X_{t}+\mu Z_{t}=\varphi
$$

where $X_{t}$ is grey derivative, $\mu$ is developing coefficient, $\varphi$ is grey action, and $Z_{t}$ is albino background value.

The parameters $\mu$ and $\varphi$ are obtained by the least square method, and the estimated parameters are substituted into the albino differential equation:

$$
\frac{\mathrm{d} Y_{t}}{\mathrm{~d} t}+\mu Y_{t}=\varphi
$$

The time response formula can be obtained by solving the above formula:

$$
\widehat{Y}_{t+1}=\left(X_{t}-\frac{\varphi}{\mu}\right) e^{-\mu t}+\frac{\varphi}{\mu} .
$$

The prediction expression of $X_{t}$ can be obtained by subtracting $\widehat{Y}_{t}$ :

$$
\widehat{X}_{t}=\widehat{Y}_{t}-\widehat{Y}_{t-1}, \quad t=2,3, \ldots, n,
$$

where $\widehat{X}_{1}=Y_{1}=X_{1}$.

The original sequence of the pull-up is the eight-dimensional sequence from 2012 to 2019. It is found that different initial values will also affect the prediction results of the prediction model. When the dimensions of the model are
$4,5,6,7$, and 8 , respectively, the model test results are shown in Table 6.

The prediction models with different initial values have passed the variance ratio test and small probability error $P$ value test. When the model dimension is 5 , the variance ratio $(0.0583)$ is the smallest, and the average relative error is $0.98 \%$, so the prediction performance of the model is the best.

Background value is used to estimate parameters $\mu$ and $\varphi$ of albino differential equation, so the optimization of background value is an important means to improve the prediction accuracy of the model. Setting different value for $\vartheta$ can optimize the background; for different value of $\vartheta$, the 5-dimensions model test results are shown in Table 7.

As shown in Table 7 , when $\vartheta=0.52$, the average relative error $(1.25 \%)$ and variance ratio $(0.0826)$ of the model are minimum.

Using the above optimal model, it can be predicted that the pull-up scores of students who will enroll in 2021 and 2022 are 2.8 and 2.6 , respectively. The prediction results of the optimal model are shown in Table 8 .

According to the trend of the actual value series and the prediction value series, with the increase of the student's enrollment year, the pull-up score shows a decreasing trend.

\section{Analysis on College Students' Physical Exercise Trend Based on LMC Model}

3.1. LMC Model Definition. The stage behavior change mode theory [20] divides the process of human behavior change into five main stages: preconsciousness stage, consciousness stage, preparation stage, behavior stage, and maintenance stage. Based on the stage behavior change mode theory, this 
TABLE 6: Model test results of different dimensions.

\begin{tabular}{lccccc}
\hline Model dimensions & Actual value in 2020 & Prediction value & Relative error (\%) & Average relative error (\%) & Variance ratio $P$ value test \\
\hline 4 & 3.27 & 3.22 & 1.5 & 1.06 & 0.0865 \\
5 & 3.27 & 3.31 & 1.2 & 0.98 & 1 \\
6 & 3.27 & 3.45 & 5.5 & 2.75 & 0.0583 \\
7 & 3.27 & 3.58 & 9.5 & 4.87 & 0.1859 \\
8 & 3.27 & 3.55 & 8.6 & 3.77 & 0.2136 \\
\hline
\end{tabular}

TABLE 7: Model test results of different $\vartheta$ in 5-dimensions model.

\begin{tabular}{lcccccc}
\hline$\vartheta$ & Actual value in 2020 & Prediction value & Relative error (\%) & Average relative error (\%) & Variance ratio & $P$ value test \\
\hline 0.35 & 3.27 & 3.16 & 3.4 & 2.75 & 0.1298 \\
0.50 & 3.27 & 3.21 & 1.8 & 1.87 & 0.0875 \\
0.52 & 3.27 & 3.22 & 1.5 & 1.25 & 0.0826 \\
0.60 & 3.27 & 3.21 & 1.8 & 1.41 & 1 \\
0.65 & 3.27 & 3.25 & 0.6 & 1.37 & 1 & 0.0865 \\
\hline
\end{tabular}

TABLE 8: The prediction results of the optimal model.

\begin{tabular}{lcccccc}
\hline Enrollment year & 2017 & 2018 & 2019 & 2020 & 2021 & 2022 \\
\hline Actual value & 4.6 & 4.2 & 3.6 & 3.3 & - & - \\
Prediction value & 4.5 & 4.2 & 3.5 & 3.2 & 2.8 & 2.6 \\
\hline
\end{tabular}

paper divides the college students into three different stages according to the exercise frequency: little, medium, and completely enough. The year is taken as a unit; based on the LMC model, the law of the number of students in the three states is studied. The basic dynamic assumptions of the LMC model are as follows:

Little state: $L(t)$ represents the number of students who exercise no more than once a week at time $t$. In the preconsciousness stage, consciousness stage, and preparation stage, all the changes are only in the consciousness level, which is difficult to distinguish from the external performance. At the same time, they have a common characteristic; that is, they have no exercise behavior. In the process of participating in physical exercise behavior, the attitude of students in the little state to physical exercise will change with the increase of the breadth and depth of knowledge. Recognizing the benefits of exercise or the acquisition of sports skills will promote the awareness of students to begin to exercise.

Medium state: $M(t)$ represents the number of students who exercise between 2 and 3 times a week at time $t$. Students in the medium state realize the importance of physical exercise and begin to have physical exercise behavior, but the low-frequency behavior is not stable, which is a potential group to form the habit of full physical exercise.

Completely enough state: $C(t)$ represents the number of students who exercise more than 3 times a week at time $t$. Students in the completely enough state have a strong sense of exercise and good executive ability. They have developed their sports behavior into a part of daily life, which will not easily change and form a greater appeal to the surrounding students.
The total number of students in a university is $N(t)$, $N(t)=L(t)+M(t)+C(t)$. The change rules [21] between states are assumed as follows:

(1) Considering a university as the whole system, the number changes among the three states in terms of years will be explored. Therefore, there are no population dynamics factors such as birth, death, and migration, but there are new students and graduates who leave school every year; the enrollment rate is denoted as $\gamma$, and the graduation rate is denoted as $\beta$.

(2) For freshmen entering the University, the high school physical education curriculum ensures that all freshmen exercise at least once a week. At the same time, a certain proportion $(1-q)$ of students will use their spare time for extra sports activities. So, at $t$ time, the number of students in the little state is $q \gamma$ $N(t)$, and the number of students in the medium state is $(1-q) \gamma N(t)$.

(3) At $t$ time, the number of students who take part in physical exercise is directly proportional to the number of students in class. Thus, the number of students moving out of the little state in unit time is proportional to $L(t)$, and the proportional coefficient is $\alpha$. In unit time, $\alpha L(t)$ students enter the medium state.

(4) Suppose that the influence of peer communication is standardized and the flow of students between the three states is gradual. At $t$ time, the number of students in the little state that can be influenced by a student with enough exercise is directly proportional to the proportion of students in the little state of the system, and the proportion coefficient is $\omega$. The number of students with a medium state added in unit time is $\omega L(t) C(t) / N(t)$. At $t$ time, the number of students in the medium state that can be affected by a student with sufficient exercise is directly proportional to the proportion of students in the medium state in the system, and the proportion coefficient is $\varepsilon$. The number of students with the 
completely enough state added in time unit time is $\varepsilon M(t) C(t) / N(t)$.

Under the above four basic assumptions, the flow chart of the transition between the three states of the proposed LMC model is shown in Figure 1.

As shown in Figure 1, the students in the little state could transit to the medium state with probability $(\alpha L+\omega L C / N)$, and the students in the medium state could transit to the completely enough state with probability $\omega M C / N$.

Let $x=L / N, y=M / N, z=C / N$, if the total population of the model remains unchanged; LMC model can be transformed into the following equivalent system:

$$
\left\{\begin{array}{l}
\frac{\mathrm{d} x}{\mathrm{~d} t}=q \gamma-\alpha x-\beta x-\omega x z, \\
\frac{\mathrm{d} y}{\mathrm{~d} t}=(1-q) \gamma+\alpha x+\omega x z-\varepsilon y z-\beta y, \\
\frac{\mathrm{d} z}{\mathrm{~d} t}=\varepsilon y z-\beta z .
\end{array}\right.
$$

3.2. Numerical Simulation and Sensitivity Analysis. In order to more intuitively observe the dynamic process of students' changes in each stage, numerical simulations are carried out in this section. The initial values are as follows: $x(0)=0.6$, $y(0)=0.3$, and $z(0)=0.1$. Other parameters are $\omega=0.9$, $\varepsilon=0.5, q=0.5$, and $\gamma=\beta=0.25$ [21]. The fitting diagram of the system is shown in Figure 2 .

It can be seen from Figure 2 that when the existing condition of positive equilibrium satisfies the inequality of $\alpha$, the system tends to a nonzero equilibrium state. When the conditions are not satisfied, the system tends to the boundary equilibrium point:

$$
R=\frac{((\gamma / \beta)-(\beta / \varepsilon))}{(q \gamma /(\alpha+\beta))}
$$

The value of threshold $R$ has a great influence on the existence and stability of the equilibrium point. Through evaluating the influence of various parameters on the threshold, we can find out the measures to increase the number of students who exercise enough. The linear influence rate $\alpha$ of threshold $R$ on classroom teaching, the proportion $q$ of freshmen without extra exercise before admission to college, and the sensitivity of influence $\varepsilon$ of the completely enough state on the medium state are discussed.

Considering that there is little change in the number of students enrolled and graduated each year [21], it is assumed that the annual enrollment rate and graduation rate of a university are equal $(\gamma=\beta)$. The sensitivity index of threshold $R$ to variable $\beta$ is defined as elasticity index:

$$
\frac{E R}{E \beta}=\frac{\partial R}{\partial \beta} \times \frac{\beta}{R}
$$

Similarly, the elasticity index of threshold $R$ for parameters $\varepsilon, \alpha$, and $q$ are as follows:

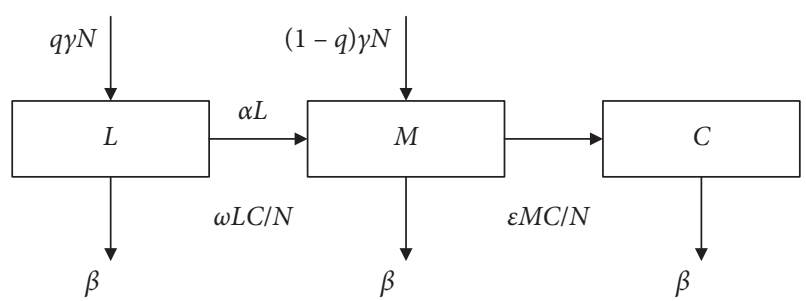

FIGURE 1: Flow chart of the transition between the three states of LMC model.

$$
\begin{aligned}
& \frac{E R}{E \varepsilon}=\frac{\partial R}{\partial \varepsilon} \times \frac{\varepsilon}{R}=\frac{\gamma \varepsilon}{\gamma \varepsilon-\beta^{2}}-1, \\
& \frac{E R}{E \alpha}=\frac{\partial R}{\partial \alpha} \times \frac{\alpha}{R}=\frac{\alpha}{\alpha+\beta}, \\
& \frac{E R}{E q}=\frac{\partial R}{\partial q} \times \frac{q}{R} .
\end{aligned}
$$

According to setting $\varepsilon=0.5, q=0.5, \gamma=\beta=0.25$ [21], it can be concluded that

$$
\left(\begin{array}{c}
\frac{E R}{E \varepsilon}<0, \quad 0<\varepsilon<0.25, \\
\frac{E R}{E \varepsilon}>1, \quad 0.25<\varepsilon<0.5, \\
0<\frac{E R}{E \varepsilon}<1, \quad 0.5<\varepsilon<1, \\
\frac{\lim \longrightarrow 0.25\left|\frac{E R}{E \varepsilon}\right|=+\infty,}{0<\frac{E R}{E \alpha}<1, \quad 0<\alpha<1,} \\
\mid \frac{E R \mid}{E q \mid}=1, \quad 0<q<1 .
\end{array}\right)
$$

It means that the effect of $\varepsilon$ on threshold $R$ is complex. Firstly, when $0<\varepsilon<0.25$, with the increase of $\varepsilon$ value, $R$ becomes smaller and negative. According to the hypothesis, students in the completely enough state mainly come from the transformation of students in the medium state. In order to get a nonzero equilibrium point, the number of new students in the completely enough state in unit time at $t$ time should be greater than the number of students who leave in unit time. That is, the value of $\varepsilon$ should be greater than 0.25 . When $\varepsilon$ approaches 0.25 from the left, $E R / E \varepsilon$ tends to be negative infinity, and when $\varepsilon$ approaches 0.25 from the right, $E R / E \varepsilon$ tends to be positive infinity. Secondly, when $0.25<\varepsilon<0.5$, with the increase of $\varepsilon$ value, the value of $E R / E \varepsilon$ is smaller and smaller, but larger than 1 . At this time, $\varepsilon$ has a very strong influence on $R$. When $0.5<\varepsilon<1$, with the increase of $\varepsilon$ value, $E R / E \varepsilon$ becomes smaller and smaller and 


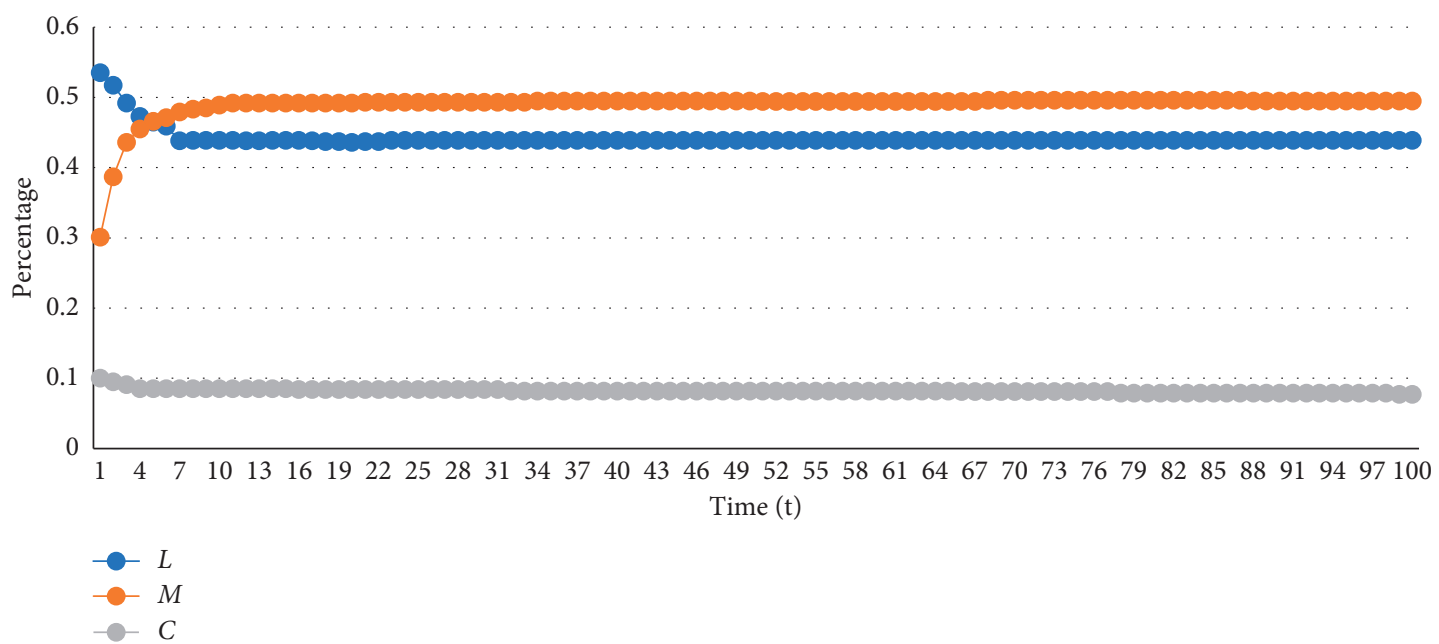

Figure 2: Fitting diagram of the system.

less than 1. When $0.5<\alpha<1, E R / E \alpha$ increases with the increase of $\varepsilon$ and is less than 1 . When $0<q<1, E R / E q$ is always -1 .

Therefore, the following conclusions can be drawn: if the influence of students in the completely enough state on students in the medium state is less than the graduation rate, there is no positive equilibrium point, and the number of students in the completely enough state will eventually tend to zero. When students in the completely enough state have less influence on students in the medium state, attention should be paid. At this time, increasing $\varepsilon$ can significantly increase the value of $R$; thus, the state of the equilibrium point can be changed. With the increasing influence of students in the completely enough state on students in the medium state, the role improvement of classroom education can have a greater effect.

In order to further determine which parameters have a great influence on the simulation results of the proposed model, the partial rank correlation coefficient (PRCC) [22] based on Latin hypercube sampling (LHS) [23] is used to analyse the parameter sensitivity of the model. PRCC measures the nonlinear but monotonic relationship between two variables and provides a measure of monotonicity between parameters. LHS is a stratified Monte Carlo sampling method, which divides the range of each parameter into $n$ equal intervals according to the distribution function of parameters and randomly selects a sample from each interval.
In this paper, the sensitivity of the simulation results $L(t)$ and $M(t)$ of the model for four parameters $q, \alpha, \omega$, and $\varepsilon$ is analysed. The sample size $n$ is set to 1000 , and the distribution and value range of each parameter are shown in Table 9.

The specific influence of parameters on different states is shown in Table 10.

The results in Table 10 show that the influence of each parameter on the final scale is very significant $(P<0.01)$ for both students in the little state and students in the completely enough state. The parameter $q$ has the greatest influence on students in the little state. The parameter $\omega$ has the greatest influence on students in the completely enough state. Table 10 shows that the relative importance of parameters can be sorted differently among different output variables. For example, the effect of $\omega$ on $L(t)$ is relatively small, while that on $C(t)$ is the largest. The correlation between parameters and different output variables is also different. $q$ was positively correlated with $L(t)$ and negatively correlated with $C(t) . \alpha, \omega$, and $\varepsilon$ were negatively correlated with $L(t)$ and positively correlated with $C(t)$.

Based on theoretical analysis, numerical simulation, and sensitivity analysis, we find that proportion of students entering the little state each year $q$, the linear influence rate of classroom teaching $\alpha$, the influence of students in the completely enough state on students in the little state $\omega$, and the influence of students in the completely enough state on students in the medium state $\varepsilon$ would affect the final stable state of the system. 
TABLE 9: PRCC analysis parameters and sampling interval.

\begin{tabular}{lcccc}
\hline Parameter & Baseline value & Variance & Distribution & Sampling interval \\
\hline$q$ & 0.8 & 0.02 & Normal distribution & $(0,1)$ \\
$\alpha$ & 0.5 & 0.02 & Normal distribution & $(0,1)$ \\
$\omega$ & 0.9 & 0.02 & Normal distribution & $(0,1)$ \\
$\varepsilon$ & 0.5 & 0.02 & Normal distribution & $(0,1)$ \\
\hline
\end{tabular}

TABLE 10: Specific influence of parameters on different states.

\begin{tabular}{|c|c|c|c|c|c|c|}
\hline \multirow{2}{*}{ Order } & \multicolumn{3}{|c|}{ Little state $(L(t))$} & \multicolumn{3}{|c|}{ Completely enough state $(C(t))$} \\
\hline & Parameter & PRCC & $P$ value & Parameter & PRCC & $P$ value \\
\hline 1 & $q$ & 0.9369 & 0 & $\omega$ & 0.9918 & 0 \\
\hline 2 & $\alpha$ & -0.8875 & 0 & $q$ & -0.8847 & $9.8523 e-306$ \\
\hline 3 & $\omega$ & -0.8869 & 0 & $\alpha$ & 0.8295 & $2.3092 e-245$ \\
\hline 4 & $\varepsilon$ & -0.5538 & $2.9161 e-83$ & $\varepsilon$ & 0.3902 & $9.6109 e-43$ \\
\hline
\end{tabular}

\section{Conclusions}

In this paper, in order to explore the current situation of college students' constitution and how to improve their physical level, two mathematical models are established based on different types of physical health data. First of all, the grey model of college physical fitness test results is established. Through correlation analysis and prediction, the factors that affect college students' pull-up performance are analysed. Then, the college students are classified according to the usual exercise frequency, and the LMC model is established to dynamically analyse the trend of college students' participation in physical exercise. The research, combined with computer simulation and mathematical model, can make people have a more in-depth and comprehensive understanding of the trend of college students' participation in physical exercise.

\section{Data Availability}

The basic data used in this paper were downloaded from the online public data set: Micro Data of College Students' Physical Health (https://bbs.pinggu.org/a-3280384.html).

\section{Conflicts of Interest}

The authors declare that they have no conflicts of interest.

\section{Acknowledgments}

This work was supported by a grant from Shandong Family Education Association of China (no. 201833).

\section{References}

[1] J. M. Sacheck, J. F. Kuder, and C. D. Economos, "Physical fitness, adiposity and metabolic risk factors in young college students," Medicine \& Science in Sports \& Exercise, vol. 42, no. 6, pp. 1039-1044, 2009.

[2] B. K. Yoon, "The level of physical fitness is an independent indicator of metabolic disease in female college students," International Journal of Human Movement Science, vol. 8, no. 2, pp. 85-95, 2014.
[3] X. Lu, C. Yang, C. Yang et al., “Test method for health-related physical fitness of college students in mobile internet environment," Mathematical Biosciences and Engineering, vol. 16, no. 4, pp. 2189-2201, 2019.

[4] B. Peng, "Analysis and intervention on the influencing factors of college students' physical fitness," Revista Brasileira de Medicina do Esporte, vol. 27, no. 9, pp. 11-13, 2021.

[5] H. Rong, X. Cheng, J. M. Garcia et al., "Survey of health literacy level and related influencing factors in military college students in Chongqing, China: a cross-sectional analysis," PLoS One, vol. 12, no. 5, Article ID e0177776, 2017.

[6] T. Noguchi, S. I. Demura, and M. Shimada, "Effect of sports club activities on the physique and physical fitness of young Japanese males," World Journal of Education, vol. 3, no. 6, pp. 27-32, 2013.

[7] E. K. White and C. S. Warren, "The influence of social anxiety on the body checking behaviors of female college students," Body Image, vol. 11, no. 4, pp. 458-63, 2014.

[8] X. Wang, "Course-taking patterns of community college students beginning in STEM: using data mining techniques to reveal viable STEM transfer pathways," Research in Higher Education, vol. 57, no. 5, pp. 544-569, 2016.

[9] Z. Kang, "Using machine learning algorithms to predict firstgeneration college students' six-year graduation: a case study," International Journal of Information Technology and Computer Science, vol. 11, no. 9, pp. 1-8, 2019.

[10] B. Sudha, A. J. Samuel, and K. Narkeesh, "Feasibility online survey to estimate physical activity level among the students studying professional courses: a cross-sectional online survey," Journal of Exercise Rehabilitation, vol. 14, no. 1, pp. 58-63, 2018.

[11] D. Bellar, L. W. Judge, and J. Petersen, "Exercise and academic performance among nursing and kinesiology students at US colleges," Journal of Education \& Health Promotion, vol. 3, no. 1, 9 pages, 2014.

[12] B. Feng, P. Zhou, and H. Dong, "Influence of long-distance running on mental health and positive sports emotions of college students," Revista Argentina de Clinica Psicologica, vol. 29, no. 2, pp. 685-691, 2020.

[13] G. Sun and S. Bin, "Router-level internet topology evolution model based on multi-subnet composited complex network model," Journal of Internet Technology, vol. 18, no. 6, pp. 1275-1283, 2017.

[14] S. Bin, G. Sun, N. Cao et al., "Collaborative filtering recommendation algorithm based on multi-relationship social 
network," Computers, Materials \& Continua, vol. 60, no. 2, pp. 659-674, 2019.

[15] D. Choszcz, R. Podstawski, and M. Wysocka-Welanc, "Measurement of motor fitness of students using the rowing ergometer," Human Movement, vol. 10, no. 1, pp. 46-52, 2009.

[16] J. Posluszny and L. A. Rubel, "The motions of an ordinary differential equation," Journal of Differential Equations, vol. 34, no. 2, pp. 291-302, 1979.

[17] Y. Wang, F. Brauer, J. Wu, and J. M. Heffernan, "A delaydependent model with HIV drug resistance during therapy," Journal of Mathematical Analysis and Applications, vol. 414, no. 2, pp. 514-531, 2014.

[18] S. Bin and G. Sun, "Optimal energy resources allocation method of wireless sensor networks for intelligent railway systems," Sensors, vol. 20, no. 2, 482 pages, 2020.

[19] C.-H. Lin, J.-L. Chen, and P.-Z. Huang, "Dissolved gases forecast to enhance oil-immersed transformer fault diagnosis with grey prediction-clustering analysis," Expert Systems, vol. 28, no. 2, pp. 123-137, 2011.

[20] A. Biehl, A. Ermagun, and A. Stathopoulos, "Utilizing multistage behavior change theory to model the process of bike share adoption," Transport Policy, vol. 77, no. 5, pp. 30-45, 2019.

[21] A. Xw, A. Lq, and T. B. Pan, "Research on college students' physical exercise trend based on compartment model," Mathematics and Computers in Simulation, vol. 180, pp. 2442, 2021.

[22] G. Sun, C.-C. Chen, and S. Bin, "Study of cascading failure in multisubnet composite complex networks," Symmetry, vol. 13, no. 3, 523 pages, 2021.

[23] G. L. Tian, S. Zhou, G. X. Sun, and C. C. Chen, "A novel intelligent recommendation algorithm based on mass diffusion," Discrete Dynamics in Nature and Society, vol. 2020, Article ID 4568171, 9 pages, 2020. 